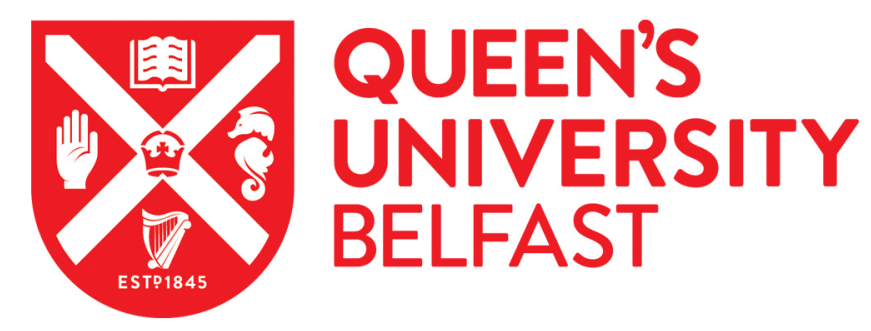

\title{
Enhanced Spread Spectrum OFDM-IM with Rotated Zadoff-Chu Sequences
}

Luong, T. V., Ko, Y., Ngo, V. D., Le, M. T., Thanh, H. L. T., \& Tran, X. N. (2019). Enhanced Spread Spectrum OFDM-IM with Rotated Zadoff-Chu Sequences. In The 5th NAFOSTED Conference on Information and Computer Science (NICS) (pp. 189-193). Institute of Electrical and Electronics Engineers Inc.. https://doi.org/10.1109/NICS.2018.8606849

Published in:

The 5th NAFOSTED Conference on Information and Computer Science (NICS)

Document Version:

Peer reviewed version

Queen's University Belfast - Research Portal:

Link to publication record in Queen's University Belfast Research Portal

Publisher rights

Copyright 2018 IEEE. This work is made available online in accordance with the publisher's policies. Please refer to any applicable terms of use of the publisher.

\section{General rights}

Copyright for the publications made accessible via the Queen's University Belfast Research Portal is retained by the author(s) and / or other copyright owners and it is a condition of accessing these publications that users recognise and abide by the legal requirements associated with these rights.

Take down policy

The Research Portal is Queen's institutional repository that provides access to Queen's research output. Every effort has been made to ensure that content in the Research Portal does not infringe any person's rights, or applicable UK laws. If you discover content in the Research Portal that you believe breaches copyright or violates any law, please contact openaccess@qub.ac.uk. 


\title{
Enhanced Spread Spectrum OFDM-IM with Rotated Zadoff-Chu Sequences
}

\author{
Thien Van Luong*, Youngwook Ko*, Vu-Duc Ngo ${ }^{\dagger \ddagger}$, Minh-Tuan Le ${ }^{\ddagger}$, Huyen Le Thi Thanh ${ }^{\S}$ and Xuan-Nam $\operatorname{Tran}^{\S}$ \\ *Institute of ECIT, Queen's University Belfast, BT3 9DT, Belfast, UK \\ ${ }^{\dagger}$ Hanoi University of Science and Technology, Hanoi, Vietnam \\ ${ }_{\ddagger}^{\ddagger}$ MobiFone R\&D Center, MobiFone Corporation, Hanoi, Vietnam \\ $\S$ Le Quy Don Technical University, 236 Hoang Quoc Viet, Cau Giay, Hanoi, Vietnam \\ Email: \{tluong01, y.ko\}@qub.ac.uk, duc.ngovu@hust.edu.vn, tuan.minh@mobifone.vn, \{huyen.ltt, \\ namtx\}@mta.edu.vn
}

\begin{abstract}
This paper proposes an enhanced scheme of spread spectrum - orthogonal frequency division multiplexing with index modulation (ESS-OFDM-IM), which employs the rotated ZadoffChu (ZC) sequences to improve the reliability of SS-OFDMIM. Particularly, unlike the conventional SS-OFDM-IM which exploits traditional ZC codes only, we design novel rotated ZC sequences for the ESS-OFDM-IM, aiming at maximizing the diversity gain of the system. We then propose a new lowcomplexity maximum likelihood (ML) detector that can achieve optimal performance at a reduced complexity compared to the ML detector. Moreover, the performance analysis is carried out to clearly show that our proposed scheme can provide the maximum diversity order of being the number of sub-carriers, which is twice larger than that of SS-OFDM-IM. Simulation results are presented to validate the superiority of our proposed scheme over not only the SS-OFDM-IM but also various existing IM schemes.
\end{abstract}

Index Terms-OFDM-IM, SS-OFDM-IM, spread spectrum, index modulation, spreading, Zadoff-Chu, low-complexity detector.

\section{INTRODUCTION}

Orthogonal frequency division multiplexing with index modulation (OFDM-IM) [1], [2] has recently emerged as a promising multicarrier modulation technique, which can achieve remarkably higher reliability and energy efficiency than the conventional OFDM. In particular, OFDM-IM activates only a subset of sub-carriers to convey information via both $M$-ary complex symbols and the indices of active subcarriers. Thus, this scheme enjoys lower transceiver complexity than the classical OFDM as it requires less modulators and demodulators. Furthermore, OFDM-IM provides an attractive trade-off between spectral efficiency (SE) and reliability, just by adjusting sub-carrier activation patterns.

Recently, various IM concepts have been proposed to improve either SE or reliability of OFDM-IM, which can be found in the survey [3]. For example, the generalized OFDMIM is proposed in [4] to increase index bits by varying the number of active sub-carriers. Also in [4], the IM notion is applied separately to in-phase and quadrature components of $M$-ary symbols to double the number of index bits. The outage probability of OFDM-IM under generalized fading channels is studied in [5]. The low-complexity greedy detector (GD) is proposed for OFDM-IM in [6] and its bit error rate (BER) is analyzed in presence of channel information state (CSI) uncertainty in [7]. A tight bound on the BER of the maximum likelihood (ML) detection is derived in [8], while a generalized framework of analyzing the symbol error probability (SEP) of both the ML and GD detectors under uncertain CSI is proposed in [9]. The combination of OFDM-IM with multiantenna systems is introduced in [10]. In [11], the impact of opportunistic transmission of OFDM-IM is investigated in terms of diversity and coding gains. The SEPs of OFDMIM with hybrid low complexity greedy detection and various diversity receptions are analyzed in [12] and then the BER of this scheme under imperfect CSI is investigated in [13]. As for the diversity issues, in [14], OFDM-IM with coordinate interleaving (CI-OFDM-IM) which can provide the diversity order of two is proposed. The repeated multicarrier index keying OFDM (MCIK-OFDM $)^{1}$ is proposed to carry the same $M$-ary symbol over all active sub-carriers, resulting in the socalled ReMO [15], [16] which also achieves the diversity order of two at the expense of SE loss. Several other IM schemes that enhance the diversity gain up to two are reported in [17], [18]. Very recently, in [19], [20], the spread OFDM-IM is proposed, in which various spreading matrices are exploited to boost the diversity order of OFDM-IM.

Another type of IM termed as code index modulation (CIM) is introduced in [21]. Different from OFDM-IM, the CIM conveys additional data bits via the indices of spreading sequences, in addition to the $M$-ary PSK/QAM modulation symbols. Notice that CIM is performed in the time-domain spreading. Meanwhile, in [22], the idea of CIM is exploited in the OFDM framework, where the spread spectrum OFDMIM (SS-OFDM-IM) is proposed by combining both IM and SS techniques. Specifically, SS-OFDM-IM selects one of the Walsh-Hadamard (WH) or Zadoff-Chu (ZC) codes to spread an $M$-ary data symbol over all sub-carriers, while additional data is carried by the index of the chosen code without any extra requirements of power or bandwidth. It is shown in [22] that SS-OFDM-IM achieves a diversity order that equals half of the number of sub-carriers.

Notice that most of the aforementioned schemes have the diversity order limited to two, which is far from the optimal

${ }^{1} \mathrm{MCIK}-\mathrm{OFDM}$ is regarded as another name of OFDM-IM. 
gain, i.e., the number of sub-carriers. To overcome this limitation, in this paper we propose an enhanced SS-OFDM-IM (ESS-OFDM-IM) by applying rotated ZC spreading sequences to OFDM-IM. Particularly, we design novel rotated ZC codes for ESS-OFDM-IM in order to maximize the diversity gain, thereby improving the reliability as compare to SS-OFDMIM with the traditional ZC or WH codes. Next, we design a new low-complexity ML detector, which can attain the optimal performance as the ML detector at much lower complexity. We then analyze the bit error probability (BEP) to clearly show that using the proposed rotated ZC codes, ESS-OFDM-IM can achieve the maximum diversity order equal to the number of sub-carriers, and thus twice larger than that of SS-OFDM-IM. As as result, our proposed scheme offers better BEP than not only SS-OFDM-IM but also various existing schemes whose diversity orders equal two.

The rest of this paper is organized as follows. Section II describes the system model, while Section III presents the proposed low-complexity detector. The performance analysis is conducted in Section IV and simulation results are provided in Section V. Finally, Section VI concludes the paper. $^{2}$

\section{SySTEM MODEL}

Consider an ESS-OFDM-IM system with $N_{c}$ sub-carriers which are partitioned into $G$ clusters, each of which contains $N$ sub-carriers, i.e., $N_{c}=G N$. Since each cluster has the same signal processing operation, without loss of generality, we address one cluster only for simplicity. In every transmission of one cluster, $p=p_{1}+p_{2}$ incoming bits are divided into two bit streams. The first $p_{1}$ bits are fed to the code selector to identify a spreading code $\mathbf{c} \in \mathcal{C}$, where $\mathcal{C}=\left\{\mathbf{c}_{1}, \ldots, \mathbf{c}_{N}\right\} \subseteq \mathbb{C}^{N \times 1}$ is the set of rotated ZC sequences to be designed below. The remaining $p_{2}$ bits are mapped to an $M$-ary complex symbol $s \in \mathcal{S}$, where $\mathcal{S}$ is the $M$-ary signal constellation.

In the proposed set $\mathcal{C}$, the first rotated $\mathrm{ZC}$ sequence $\mathbf{c}_{1}=$ $\left[c_{1}, \ldots, c_{N}\right]^{T}$ is the $\mathrm{ZC}$ sequence [23] defined as follows

$$
c_{n}=\left\{\begin{array}{ll}
e^{-\frac{j 2 \pi k}{N}\left(\frac{n^{2}}{2}+b n\right)} & \text { for even } N \\
e^{-\frac{j 2 \pi k}{N}\left[\frac{n(n+1)}{2}+b n\right]} & \text { for odd } N
\end{array},\right.
$$

where $n=1,2, \ldots, N, b$ is any integer and $k$ is any integer relatively prime to $N$. The $n$-th rotated code $\mathbf{c}_{n}$ for $n \geq 2$ is given as the $n$-th cyclically shifted version of $\mathbf{c}_{1}$ rotated by an angle $e^{\frac{j 2 \pi(n-1)}{D}}$, where $D$ is chosen to be an integer greater and relatively prime to both $N$ and $M$. When $N=2^{p_{1}}$, we can simply choose $D=\max \{M, N\}+1$ to make elements of all codes different from each other, and in other cases of $N$, we can select $D=M N+1$. For example, for $n=2$ we obtain $\mathbf{c}_{2}=e^{\frac{j 2 \pi}{D}}\left[c_{N}, c_{1}, \ldots, c_{N-1}\right]^{T}$. Though simple, this approach can significantly improve the diversity gain of ESSOFDM-IM, compared with SS-OFDM-IM, as shown in the performance analysis afterwards.

\footnotetext{
${ }^{2}$ Notation: Upper-case bold and lower-case bold letters are used for matrices and vectors, respectively. $(.)^{T}$ and $(.)^{H}$ stand for the transpose operation and the Hermitian operation, respectively. $\|$.$\| denotes the Frobenious norm. The$ floor function is denoted by [.]. $\mathcal{C N}\left(0, \sigma^{2}\right)$ represents the complex Gaussian distribution with zero mean and variance $\sigma^{2}$. The ring of complex number is denoted by $\mathbb{C}$.
}

Unlike the $\mathrm{WH}$ codes, the length of the $\mathrm{ZC}$ is more flexible, which can be any positive integer, leading to $p_{1}=\left\lfloor\log _{2} N\right\rfloor$. Thus, for given $N$ and $M$, the data rate of the system is

$$
R=\frac{\left\lfloor\log _{2} N\right\rfloor+\log _{2} M}{N}(\mathrm{bps} / \mathrm{Hz})
$$

After determining $\mathbf{c}$ and $s$ based on the incoming bits, the transmitted vector $\mathbf{x}$ is obtained by spreading $s$ across $N$ sub-carriers, using $\mathbf{c}$, i.e., $\mathbf{x}=\mathbf{c} s$. Then, $\mathbf{x}$ is processed by the inverse fast Fourier transform (IFFT). At the receiver, the received signal vector in the frequency domain is given by

$$
\mathbf{y}=\mathbf{H x}+\mathbf{n}=\mathbf{H} \mathbf{c} s+\mathbf{n},
$$

where $\mathbf{H}=\operatorname{diag}\left\{h_{1}, \ldots, h_{N}\right\}$ denotes the Rayleigh fading channel matrix with $h_{n} \sim \mathcal{C N}(0,1)$ and $\mathbf{n}$ is the additive white Gaussian noise vector with its entries $\sim \mathcal{C N}\left(0, N_{0}\right)$.

For signal detection, the maximum likelihood (ML) detector can be used to attain the optimal performance as follows

$$
(\hat{s}, \hat{\mathbf{c}})=\arg \min _{s, \mathbf{c}}\|\mathbf{y}-\mathbf{H} \mathbf{c} s\|^{2} .
$$

Since the ML is not practical when system parameters such as $N, M$ increase. In [22], the low complexity maximal ratio combining (MRC) detector is proposed for SS-OFDM-IM. However, the MRC does not perform well in our proposed scheme. Therefore, a novel low-complexity ML detector is developed in the following section to resolve the issue.

\section{LOW COMPLEXITY ML DETECTOR}

In this section, the proposed ML detector is presented. Then its computational complexity is evaluated and compared with both the ML and the MRC.

\section{A. Proposed Detector}

The proposed low-complexity ML (lowML) detector first estimates the $M$-ary data symbol $s_{n}$ for each spreading sequence $c_{n} \in \mathcal{C}$, utilizing the MRC approach, as follows

$$
s_{n}=\mathcal{D}\left\{\mathbf{H}_{n}^{H} \mathbf{y} / T\right\},
$$

where $T=\|\mathbf{H}\|^{2}, \mathbf{H}_{n}=\mathbf{H} \mathbf{c}_{n}$ for $n=1, \ldots, 2^{p_{1}}$, and $\mathcal{D}(s)$ denotes the digital demodulator function that returns the $M$ ary symbol being closest to $s$. Then, using $s_{n}$, we calculate the distance $\Delta_{n}=\left\|\mathbf{y}-\mathbf{H}_{n} s_{n}\right\|^{2}$. Finally, the transmitted $M$-ary symbol and spreading code are respectively recovered by

$$
\hat{s}=s_{\hat{n}}, \hat{\mathbf{c}}=\mathbf{c}_{\hat{n}},
$$

where $\hat{n}=\arg \min _{n=1, \ldots, 2^{p_{1}}} \Delta_{n}$. The proposed lowML detector can be summarized in Algorithm 1.

It is worth noting that the lowML detector obviously does not involve any approximation or assumption, thereby exhibiting the same optimal performance as the ML detector. In addition, while the MRC separately estimates the spreading code and the $M$-ary symbol, the lowML jointly decodes both these signal dimensions. Hence, our proposed detector can provide better performance than the MRC at the cost of higher computational complexity. 


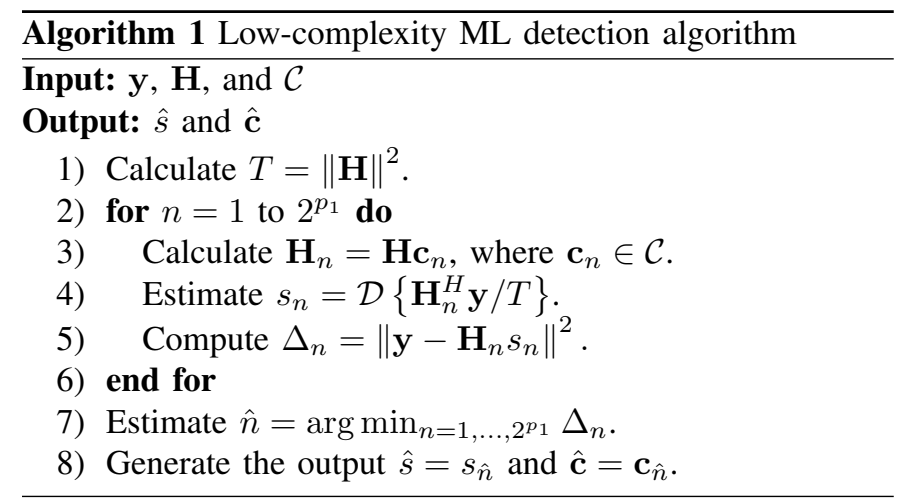

\section{B. Complexity Analysis and Comparison}

We evaluate the computational complexity of the proposed detector with respect to the number of floating point operations (flops) per sub-carrier. Notice that a flop can be counted as a real summation, a real division, a real multiplication or a real square root. For example, a complex multiplication contains 4 real multiplications and 2 real additions, thus is considered as 6 flops. We also compute the complexities of the ML and the MRC for comparison.

For simplicity in the calculation, we assume $N=2^{p_{1}}$. According to Algorithm 1, numbers of flops involved in steps $1,3,4$ and 5 are approximately $4 N, 6 N, 8 N$ and $12 N$, respectively. As a result, the complexity of the proposed detector is $28 N+4$ (flops/sub-carrier), which is the order of $\mathcal{O}(N)$. Here, it should be noted that since the complexity of the digital demodulator function in step 4 is negligible in comparison with other calculations, the complexity of the proposed detector is roughly independent of the modulation size $M$. Similarly, the complexities of the ML and the MRC are approximated by $18 M N$ and $14 N+15$ (flops/sub-carrier), respectively, and their corresponding orders are $\mathcal{O}(M N)$ and $\mathcal{O}(N)$. It is clear that the complexity of the ML detector relies on both $M$ and $N$, while that of the proposed lowML, similar to the MRC, depends almost on $N$ only.

The complexity comparison among three detectors for various values of $N$ and $M$ is depicted in Table I. It can be seen that the complexity of the proposed detector is much lower than that of the ML, while it is slightly higher than the MRC. For example, when $(N, M)=(4,64)$, the proposed lowML can save $97.5 \%$ computational complexity compared to the ML, while the reduction of the MRC is $98.5 \%$.

TABLE I

COMPLEXITY COMPARISON AMONG THE PROPOSED DETECTOR, THE ML AND THE MRC UNDER VARIOUS CONFIGURATIONS $(N, M)$

\begin{tabular}{|c|c|c|c|c|c|}
\hline$(N, M)$ & $(4,4)$ & $(4,64)$ & $(4,512)$ & $(8,32)$ & $(8,1024)$ \\
\hline \hline ML & 288 & 4608 & 36864 & 4608 & 147456 \\
\hline MRC [22] & 71 & 71 & 71 & 127 & 127 \\
\hline Proposed & 116 & 116 & 116 & 228 & 228 \\
\hline
\end{tabular}

\section{PERformance AnAlysis}

We analytically evaluate the pairwise error probability (PEP) of ESS-OFDM-IM to show the enhancement of the diversity gain when employing the proposed rotated $\mathrm{ZC}$ sequences. Then, the upper bound on the BEP is derived.

The conditional PEP that the transmitted $\mathbf{x}=\mathbf{c} s$ is erroneously detected as $\hat{\mathbf{x}}=\hat{\mathbf{c}} \hat{s} \neq \mathbf{x}$, for given $\mathbf{H}$, is given by

$$
P(\mathbf{x} \rightarrow \hat{\mathbf{x}} \mid \mathbf{H})=Q\left(\sqrt{\frac{\|\mathbf{H}(\mathbf{c} s-\hat{\mathbf{c}} \hat{s})\|^{2}}{2 N_{0}}}\right),
$$

where $Q($.$) represents the Gaussian tail probability. We as-$ sume that $N_{0}=1 / \bar{\gamma}$ where $\bar{\gamma}$ is the average signal-to-noise ratio (SNR) per sub-carrier, $\mathbf{c}=\left[c_{1}, \ldots, c_{N}\right]^{T}, \hat{\mathbf{c}}=\left[\hat{c}_{1}, \ldots, \hat{c}_{N}\right]^{T}$ and $\kappa_{n}=\left|c_{n} s-\hat{c}_{n} \hat{s}\right|^{2}$ for $n=1, \ldots, N$. Then, (7) can be rewritten as

$$
P(\mathbf{x} \rightarrow \hat{\mathbf{x}} \mid \mathbf{H})=Q\left(\sqrt{\frac{\sum_{n=1}^{N} \kappa_{n} \gamma_{n}}{2}}\right),
$$

where $\gamma_{n}=\bar{\gamma}\left|h_{n}\right|^{2}$ is the instantaneous SNR at the $n$-th subcarrier. Using the approximation of Q-function as $Q(x) \approx$ $e^{-x^{2} / 2} / 12+e^{-2 x^{2} / 3} / 4$, (8) can be approximated as

$$
P(\mathbf{x} \rightarrow \hat{\mathbf{x}} \mid \mathbf{H}) \approx \frac{1}{12} e^{-\frac{\Lambda}{4}}+\frac{1}{4} e^{-\frac{\Lambda}{3}},
$$

where $\Lambda=\sum_{n=1}^{N} \kappa_{n} \gamma_{n}$. Note that under Rayleigh fading channel model, the moment generating function (MGF) of $\Lambda$ is given by $\mathcal{M}_{\Lambda}(t)=\prod_{n=1}^{N}\left(1-\kappa_{n} \bar{\gamma} t\right)$. Here, applying the MGF approach to (9), the unconditional PEP is obtained as

$$
P(\mathbf{x} \rightarrow \hat{\mathbf{x}}) \approx \frac{1 / 12}{\prod_{n=1}^{N}\left(1+\frac{\kappa_{n} \bar{\gamma}}{4}\right)}+\frac{1 / 4}{\prod_{n=1}^{N}\left(1+\frac{\kappa_{n} \bar{\gamma}}{3}\right)}
$$

It can be seen from (10) that the diversity order of the PEP is the number of non-zero elements $\kappa_{n}$. To determine the diversity order of the proposed scheme, we consider three following possible cases of PEP error events $\mathbf{x} \rightarrow \hat{\mathbf{x}}$. Firstly, $\mathbf{c}=\hat{\mathbf{c}}$ and $s \neq \hat{s}$, leading to $\kappa_{n}=|s|^{2} \neq 0$ for every $n$, thus the diversity order in this case is $N$.

Secondly, $\mathbf{c} \neq \hat{\mathbf{c}}$ and $s=\hat{s}$, we obtain $\kappa_{n}=|s|^{2}\left|c_{n}-\hat{c}_{n}\right|^{2}$. Thus, the diversity order becomes the Hamming distance between two different codes, which is proved to be $N$ as follows. When $N$ is even, it is assumed from (1) that $c_{n}=$ $e^{-\frac{j \pi m k_{1}^{2}}{N}+\frac{j 2 \pi q_{1}}{D}}$ and $\hat{c}_{n}=e^{-\frac{j \pi m k_{2}^{2}}{N}+\frac{j 2 \pi q_{2}}{D}}$, where $0 \leq q_{1}<$ $q_{2}<N$ and $1 \leq k_{1}, k_{2} \leq N$. Here, we select $k=1$ and $b=0$ in (1) to define such $c_{n}$ and $\hat{c}_{n}$. If $c_{n}=\hat{c}_{n}$, we attain

$$
\operatorname{Dm}\left(k_{2}^{2}-k_{1}^{2}\right)=2\left(q_{2}-q_{1}\right) N
$$

Since $D$ is relatively prime to both 2 and $N$, it is obtained from $(11)$ that $q_{2}-q_{1} \equiv 0(\bmod D)$. However, this conflicts with the fact of $0<q_{2}-q_{1}<D$. Hence, $c_{n} \neq \hat{c}_{n}$ for every $n$, which leads to the diversity order in this case is $N$. Notice that for odd $N$, we also arrive such the same conclusion.

In the last case of $\mathbf{c} \neq \hat{\mathbf{c}}$ and $s \neq \hat{s}$, we assume $s=e^{\frac{j 2 \pi l_{1}}{M}}$ and $\hat{s}=e^{\frac{j 2 \pi l_{2}}{M}}, 0 \leq l_{1}<l_{2}<M$ when the $M$-ary PSK modulation is used. Note that $c_{n} s-\hat{c}_{n} \hat{s}=0$ is equivalent to

$$
D\left[2\left(l_{1}-l_{2}\right)+M m\left(k_{2}^{2}-k_{1}^{2}\right)\right]=2\left(q_{2}-q_{1}\right) M N .
$$

Applying the same approach as in the second case to (12), it is shown that $\kappa_{n}$ is always non-zero for every $n$, leading to the diversity order achieved in this case is still $N$. 
Remark 1: In conventional SS-OFDM-IM which relies on traditional $\mathrm{ZC}$ or $\mathrm{WH}$ codes, there always exists two distinct codes so that their Hamming distance is $N / 2$. This makes the diversity order of SS-OFDM-IM in the second case above always limited to $N / 2$. By contrast, as analyzed above, applying the proposed rotated ZC codes, ESS-OFDMIM ensures to achieve the maximum diversity gain, i.e., $N$. As a consequence, the proposed scheme can achieve significantly higher reliability than SS-OFDM-IM as well as other IM schemes as will be validated in Section V.

Remark 2: Utilizing (10), we obtain the upper bound on the BEP of ESS-OFDM-IM, based on the union bound theory, as

$$
P_{b} \leq \frac{1}{p M N} \sum_{\mathbf{x}} \sum_{\hat{\mathbf{x}}} P(\mathbf{x} \rightarrow \hat{\mathbf{x}}) \beta(\mathbf{x}, \hat{\mathbf{x}}),
$$

$\beta(\mathbf{x}, \hat{\mathbf{x}})$ is the number of bit errors in the error event $(\mathbf{x} \rightarrow \hat{\mathbf{x}})$.

\section{Simulation Results}

In this section, simulation results are presented to validate the error performance of the proposed ESS-OFDM-IM as well as lowML detector. We compare our scheme with OFDMIM and several recent IM schemes such as SS-OFDM-IM [22], ReMO [16] and CI-OFDM-IM [14]. The configurations of ReMO, CI-OFDM-IM and OFDM-IM are denoted as $(N, K, M)$ where $N$ and $M$ are the same as the proposed scheme, while $K$ is the number of active sub-carriers.

Fig. 1. compares the BEP of ESS-OFDM-IM with SSOFDM-IM and OFDM-IM, at the data rate of $1.5 \mathrm{bps} / \mathrm{Hz}$, when $N=2, M=4$ and $K=1$. Since ReMO and CIOFDM-IM require $K \geq 2$, they do not work in this case. The proposed scheme employs three detectors including the ML, MRC [22] and the proposed lowML detector, while others use the ML. As seen via Fig. 1, ESS-OFDM-IM is superior to the benchmarks in terms of the BEP performance. For example, at $\mathrm{BEP}=10^{-4}$, our scheme achieves SNR gains of $9 \mathrm{~dB}$ and $12 \mathrm{~dB}$ over OFDM-IM and SS-OFDM-IM, respectively. This is because using the proposed rotated ZC sequences, ESSOFDM-IM attains the maximum diversity order, which is 2, i.e., $N$ as proved in Section IV, while both SS-OFDM-IM and OFDM-IM only provide the diversity order of one. Moreover, the proposed lowML detector exhibits the optimal BEP as the ML. Meanwhile, compared to the MRC, our detector has the considerably better BEP performance. Fig. 1 also confirms the tightness of the derived upper bound on the BEP of ESSOFDM-IM, especially when the SNRs get higher.

In Fig. 2, the BEP comparison among ESS-OFDM-IM, SS-OFDM-IM, ReMO, CI-OFDM-IM and OFDM-IM is illustrated, at the data rate of $1 \mathrm{bps} / \mathrm{Hz}$, when $N=4$, $M=\{2,4\}$ and $K=\{1,2\}$. Once again, a significant BEP improvement of the proposed scheme can be seen from Fig. 2 in comparison with its benchmark schemes, especially at the increasing SNRs. Particularly, this figure clearly shows that our scheme can provide the diversity order of four, while the benchmark schemes only offer the diversity orders of two or one. For instance, at BEP $=10^{-5}$, there are remarkable SNR gains of approximately 5, 7, 8 and $20 \mathrm{~dB}$ achieved by ESS-OFDM-IM over SS-OFDM-IM, ReMO, CI-OFDM-IM

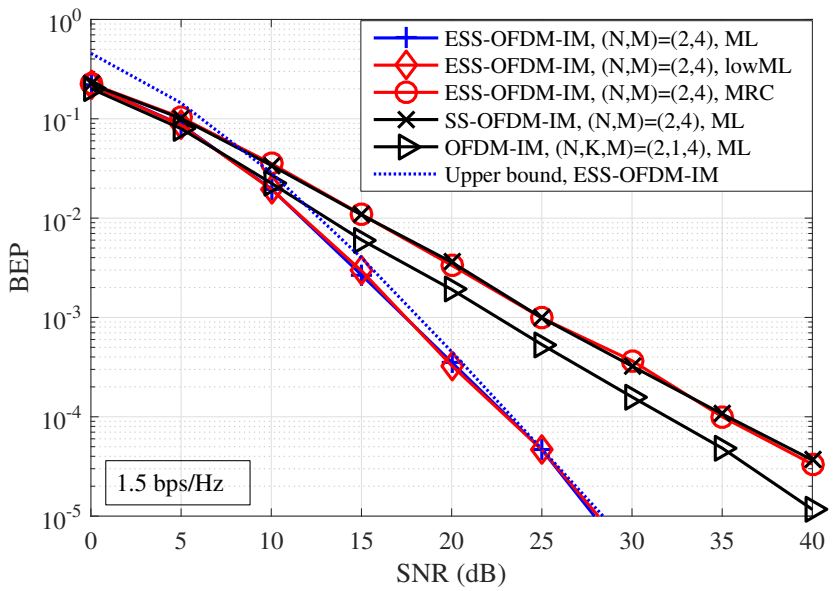

Fig. 1. BEP comparison among ESS-OFDM-IM, SS-OFDM-IM and OFDM$\mathrm{IM}$ at the data rate $1.5 \mathrm{bps} / \mathrm{Hz}$, when $N=2, M=4$ and $K=1$.

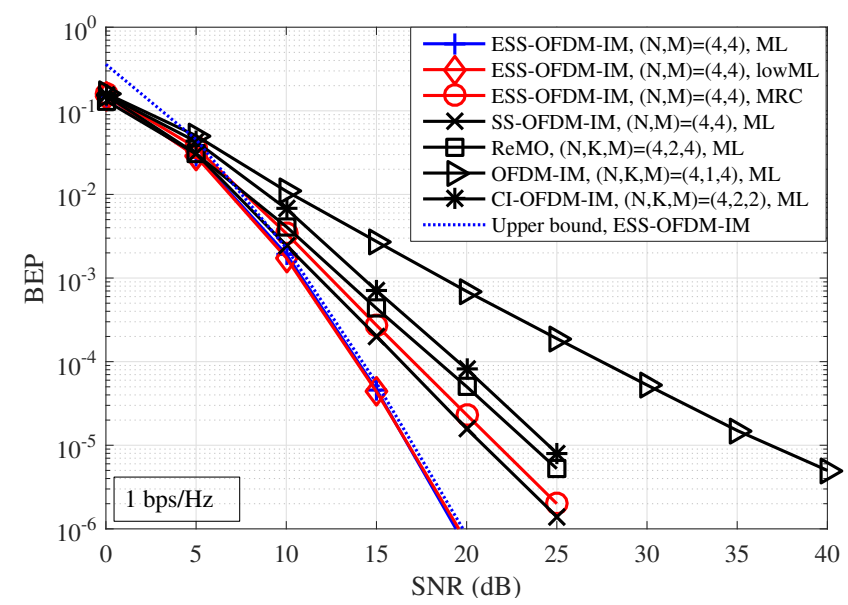

Fig. 2. BEP comparison among ESS-OFDM-IM, SS-OFDM-IM, ReMO, CIOFDM-IM and OFDM-IM at the data rate $1 \mathrm{bps} / \mathrm{Hz}$, when $N=4, M=$ $\{2,4\}$ and $K=\{1,2\}$.

and OFDM-IM, respectively. In addition, Fig. 2 once again clearly demonstrates the superiority of the proposed detector over the MRC detector.

\section{CONCLUSION}

We have proposed an enhanced SS-OFDM-IM scheme, which employs the rotated $\mathrm{ZC}$ codes to improve the reliability over SS-OFDM-IM exploiting the traditional ZC or WH codes. In particular, we provided a novel method to design the rotated $\mathrm{ZC}$ codes that can maximize the diversity order. The BEP performance was analyzed to clearly show that employing the proposed rotated ZC codes, ESS-OFDM-IM achieves the maximum diversity order which is twice larger than that of SS-OFDM-IM. We also derived a novel lowML detector that exhibits the optimal performance as the ML, at the much lower complexity. Specifically, unlike the ML, the lowML detector has the complexity which is roughly independent of the modulation size. Simulation results clearly show that our proposed scheme significantly outperforms the benchmarks. Furthermore, the proposed detector performs considerably better than the MRC at slightly higher complexity. 


\section{REFERENCES}

[1] R. Abu-alhiga and H. Haas, "Subcarrier-index modulation OFDM," in Proc. IEEE Pers., Indoor., Mobile Radio Commun., Sept 2009, pp. 177181.

[2] E. Basar, U. Aygolu, E. Panayirci, and H. V. Poor, "Orthogonal frequency division multiplexing with index modulation," IEEE Trans. Signal Process., vol. 61, no. 22, pp. 5536-5549, Nov 2013.

[3] E. Basar, M. Wen, R. Mesleh, M. D. Renzo, Y. Xiao, and H. Haas, "Index modulation techniques for next-generation wireless networks," IEEE Access, vol. 5, pp. 16693-16746, 2017.

[4] R. Fan, Y. J. Yu, and Y. L. Guan, "Generalization of orthogonal frequency division multiplexing with index modulation," IEEE Trans. Wireless Commun., vol. 14, no. 10, pp. 5350-5359, Oct 2015.

[5] T. V. Luong and Y. Ko, "Symbol error outage performance analysis of MCIK-OFDM over complex TWDP fading," in Proc. Eur. Wireless, May 2017, pp. 1-5

[6] J. Crawford and Y. Ko, "Low complexity greedy detection method with generalized multicarrier index keying OFDM," in Proc. IEEE Pers., Indoor., Mobile Radio Commun., Aug 2015, pp. 688-693.

[7] T. V. Luong and Y. Ko, "A tight bound on BER of MCIK-OFDM with greedy detection and imperfect CSI," IEEE Commun. Lett., vol. 21, no. 12 , pp. 2594 - 2597, Dec 2017.

[8] Y. Ko, "A tight upper bound on bit error rate of joint OFDM and multicarrier index keying," IEEE Commun. Lett., vol. 18, no. 10, pp. 1763 1766, Oct 2014.

[9] T. V. Luong and Y. Ko, "Impact of CSI uncertainty on MCIK-OFDM: tight, closed-form symbol error probability analysis," IEEE Trans. Veh. Technol., vol. 67, no. 2, pp. 1272 - 1279, Feb 2018.

[10] E. Basar, "On multiple-input multiple-output OFDM with index modulation for next generation wireless networks," IEEE Trans. Signal Process., vol. 64, no. 15, pp. 3868-3878, Aug 2016.

[11] T. V. Luong and Y. Ko, "Impact of oppotunistic transmission on MCIKOFDM: Diversity and coding gains," in Proc. 4th EAI INISCOM, Aug 2018.

[12] J. Crawford, E. Chatziantoniou, and Y. Ko, "On the SEP analysis of OFDM index modulation with hybrid low complexity greedy detection and diversity reception," IEEE Trans. Veh. Technol., vol. 66, no. 9, pp. 8103-8118, Sept 2017.

[13] T. V. Luong and Y. Ko, "The BER analysis of MRC-aided greedy detection for OFDM-IM in presence of uncertain CSI," IEEE Wireless Commun. Lett., vol. 7, no. 4, pp. 566-569, Aug 2018.

[14] E. Basar, "OFDM with index modulation using coordinate interleaving," IEEE Wireless Commun. Lett., vol. 4, no. 4, pp. 381-384, Aug 2015.

[15] T. V. Luong and Y. Ko, "A closed-form symbol error probability for MCIK-OFDM with frequency diversity," in Proc. IEEE SPAWC, Jul 2017, pp. 1-5.

[16] T. V. Luong, Y. Ko, and J. Choi, "Repeated MCIK-OFDM with enhanced transmit diversity under CSI uncertainty," IEEE Trans. Wireless Commun., vol. 17, no. 6, pp. 4079-4088, June 2018.

[17] M. Wen, B. Ye, E. Basar, Q. Li, and F. Ji, "Enhanced orthogonal frequency division multiplexing with index modulation," IEEE Trans. Wireless Commun., vol. 16, no. 7, pp. 4786 - 4801, July 2017.

[18] J. Zheng and R. Chen, "Achieving transmit diversity in OFDM-IM by utilizing multiple signal constellations," IEEE Access, vol. 5, no. 99, pp. 8978 - 8988, 2017.

[19] T. V. Luong and Y. Ko, "Spread OFDM-IM with precoding matrix and low-complexity detection designs," IEEE Trans. Veh. Technol., to be published.

[20] T. V. Luong, Y. Ko, and J. Choi, "Precoding for spread OFDM IM," in Proc. IEEE 87th Veh. Technol. Conf. (VTC Spring), 2018, pp. 1-5.

[21] G. Kaddoum, Y. Nijsure, and H. Tran, "Generalized code index modulation technique for high-data-rate communication systems," IEEE Trans. Veh. Technol., vol. 65, no. 9, pp. 7000-7009, Sept 2016.

[22] Q. Li, M. Wen, E. Basar, and F. Chen, "Index modulated OFDM spread spectrum," IEEE Trans. Wireless Commun., vol. 17, no. 4, pp. 2360 2374, 2018.

[23] D. Chu, "Polyphase codes with good periodic correlation properties (corresp.)," IEEE Trans. Inf. Theory, vol. 18, no. 4, pp. 531-532, July 1972. 